\title{
The complex of maximal lattice free simplices
}

\author{
Imre Bárány ${ }^{\mathrm{a}}$, Roger Howe ${ }^{\mathrm{b}}$, Herbert E. Scarf ${ }^{\mathrm{c}, *}$ \\ ${ }^{a}$ Mathematical Institute, Budapest, P.O.B. 127, 1364, Hungary \\ ${ }^{\mathrm{b}}$ Department of Mathematics, Yale University, New Haven, CT 06520, USA \\ 'Department of Economics, Yale University, New Haven, CT 06520, USA
}

Received 4 January 1993; revised manuscript received 3 January 1994

\begin{abstract}
The simplicial complex $K(A)$ is defined to be the collection of simplices, and their proper subsimplices, representing maximal lattice free bodies of the form $(x: A x \leqslant b)$, with $A$ a fixed generic $(n+1) \times n$ matrix. The topological space associated with $K(A)$ is shown to be homeomorphic to $\mathbb{R}^{n}$, and the space obtained by identifying lattice translates of these simplices is homeorphic to the $n$-torus.
\end{abstract}

Keywords: Minimal test sets for integer programming; Simplicial complexes; Maximal lattice free bodies

\section{Introduction}

We study the global topological structure of a simplicial complex arising naturally in the study of integer programming. The simplices in the complex consist of maximal lattice free bodies defined in the following way. Let $Z^{n}$ be the lattice of integers in $\mathbb{R}^{n}$, and let $A$ be an $(n+1) \times n$ matrix, with $i$ th row denoted by $a_{i}$, and which satisfies the two conditions:

(A1) There is a unique (up to a positive multiple) strictly positive $(n+1)$ row-vector $\pi$ such that $\pi A=0$, and

(A2) If, for any index $i, a_{i} \cdot z=0$ for some integral $z$, then $z=0$. Condition (A1) implies that the $n \times n$ minors of $A$ are non-singular and that for any $b$ the simplex $(x \mid A x \leqslant b)$ is bounded. Since, for our purposes, the rows of $A$ can be normalized arbitrarily, there is no loss in generality in assuming that $\pi=1=(1, \ldots, 1)$. Condition (A2) asserts that for any

\footnotetext{
*Corresponding author.

The first author was partially supported by Hungarian NSF grants 1907 and 1909, and also by U.S. NSF grant CCR-9111491. The research of the second author was supported by DMS 9103608 and the third author by NSF grant SES9121936.
} 
$b_{i}$ the hyperplane $a_{i} \cdot x=b_{i}$ contains at most one lattice point in $Z^{n}$. As it will turn out, this condition can be relaxed so that small perturbations of $A$ are also admissible.

A maximal lattice free body is a body of the form $K=(x \mid A x \leqslant b)$ containing no lattice points in its interior, and such that any closed convex body which properly contains $K$ does have a lattice point in its interior. It follows from (A2) that for each $i$ there is a single lattice point $z^{i}$ on the hyperplane $a_{i} \cdot x=b_{i}$, that these lattice points are distinct for different $i$, and that $a_{i} \cdot z^{j}<a_{i} \cdot z^{i}$ for $j \neq i$. Moreover there is no lattice point $z$ such that $a_{i} \cdot z<a_{i} \cdot z^{i}$ for $i=0$, $1, \ldots, n$.

The abstract simplicial complex $K(A)$ consists of all of the formal simplices $\left(z^{0}, z^{1}, \ldots\right.$, $z^{n}$ ), oriented in this fashion, and all of their subsimplices. The complex contains an infinite number of simplices, since an arbitrary lattice translate of a maximal lattice free body is also maximal lattice free. It will be shown, however, that (A1) implies that the complex is locally finite in the sense that every lattice point $z$ is a vertex of a finite number of such simplices (White, 1983). It can also be shown that each $(n-1)$ face $\left(z^{1}, \ldots, z^{i-1}, z^{i+1}\right.$, $\ldots, z^{n}$ ) is incident to precisely two $n$-simplices in the complex, with opposite derived orientations, so that the complex is an orientable pseudo-manifold (Scarf, 1981a).

The simplicial complex $K(A)$, being locally finite, can be provided with the usual topology: Give each simplex in $K(A)$ its customary topology, and realize $K(A)$ as the disjoint union of its vertices and the relative interiors of its simplices and subsimplices. A set in the union is defined to be closed if its intersection with the closure of each simplex in $K(A)$ is a closed set. The associated topological space will be written as $|K(A)|$.

When $n=2$, there is a basis, $b^{1}, b^{2}$, for the lattice $Z^{2}$, such that the simplices in $K(A)$ are the lattice translates of $\left(0, b^{1}, b^{1}+b^{2}\right)$ and $\left(0, b^{2}, b^{1}+b^{2}\right)$, thereby providing a simple geometric realization of the abstract complex as $\mathbb{R}^{2}$. When $n \geqslant 3$, however, the convex hulls of two distinct $n$-simplices may contain common interior points, and the global structure of the complex is no longer transparent. Some indication of this structure is suggested by the following result which also appears in White (1983).

Assume that the rows of $A$ are permuted so that $\operatorname{det}\left[1^{\prime}, A\right]>0$, and for each simplex $S=\left(z^{0}, z^{1}, \ldots, z^{n}\right)$ in $K(A)$, define

$$
\operatorname{index}(S)=\operatorname{sign}\left[\operatorname{det}\left(z^{1}-z^{0}, \ldots, z^{n}-z^{0}\right)\right] \text {, }
$$

or zero if the determinant vanishes. Then for generic $x \in \mathbb{R}^{n}$,

$$
\sum(\operatorname{index}(S) \mid x \in \operatorname{convex}(S))=1 .
$$

The result suggests that the natural geometric realization of the complex $K(A)$ by means of the convex hulls of its simplices may be an intricate folding of $\mathbb{R}^{n}$ into itself; and this is, in fact, our major conclusion.

Theorem 1. $|K(A)|$ is homeomorphic to $\mathbb{R}^{n}$.

The group of integers $Z^{n}$ acts on $K(A)$, since the lattice translate of a simplex in $K(A)$ is also a simplex in $K(A)$. We may, therefore, obtain a new complex, $K Z(A)$, by identifying 
those simplices or subsimplices of $K(A)$, which are lattice translates of each other. Local finiteness of $K(A)$ implies that the new complex has a finite number of simplices of each dimension $j=0, \ldots, n$. In particular $K Z(A)$ has a single vertex. $K Z(A)$ is not a conventional simplicial complex, but is a more general object known as a CW complex (Massey, 1980).

$K Z(A)$ is realized as the disjoint union of a single vertex and the relative interiors of the simplices and subsimplices of $K(A)$, with lattice translates identified. As before, a set in the union is closed if its intersection with the closure of any simplex is a closed set. The topological space obtained in this fashion is denoted by $|K Z(A)|$, and is clearly homeomorphic to $|K(A)| / Z^{n}$.

White (1983) contains an additional result, bearing on the structure of $K Z(A)$. Again assume that the rows of $A$ are permuted so that $\operatorname{det}\left[\mathbf{1}^{\prime}, A\right]>0$.

Then

$$
\sum\left[\operatorname{det}\left(z^{1}-z^{0}, \ldots, z^{n}-z^{0}\right)\right] / n !=1,
$$

with the sum taken over a set of representatives in $K(A)$ of the maximal simplices in $K Z(A)$. The result states that the sum of the signed volumes of the simplices in $K Z(A)$ is equal to unity, and is consistent with the following theorem, conjectured by Lex Schrijver.

\section{Theorem 2. $|K Z(A)|$ is homeomorphic to the n-torus.}

The demonstration of Theorem 1 involves the construction of a simplicial mapping of the vertices of $K(A)$ into $\mathbb{R}^{n+1}$, such that the convex hulls of the images of the vertices of two distinct $n$-simplices no longer overlap. They form, in fact, the facets of a convex set in $\mathbb{R}^{n+1}$.

Before presenting the details of this construction it may provide motivation for the study of $K(A)$ to say a few words about the relationship between maximal lattice free bodies and integer programming. Consider the integer program

$$
\begin{array}{ll}
\min & \sum a_{0, j} \cdot z_{j} \\
\text { subject to } & \sum a_{i, j} \cdot z_{j} \leqslant b_{i}, \quad i=1, \ldots, n \text { and } z \in Z^{n} .
\end{array}
$$

Let $N(0)$ be an arbitrary set of lattice points which is symmetric about the origin. For a particular right-hand side $b$, a lattice point $z$ is said to be a local minimum with respect to $N(0)$ if $z$ is a feasible solution to (1), and if, for every $h \in N(0), z+h$ is either infeasible or yields a higher value of the objective function $\sum a_{0, j} \cdot\left(z_{j}+h_{j}\right)$.

Under assumptions (A1) and (A2), it may be demonstrated that there is a unique minimal $N(0)$, called the set of neighbors of the origin associated with the matrix $A$, for which a local minimum with respect to $N(0)$ is a global minimum for each integer program obtained by specifying a right hand side $b$. The set of neighbors may be shown to consist of precisely those lattice points $h$ which are contained in some simplex of $K(A)$, another of whose vertices is the origin (Scarf, 1986). Alternatively, a lattice point $h$ is contained in $N(0)$ iff the only lattice points in the body 
$(x \mid A x \leqslant b), \quad$ with $b_{i}=\max \left(0, a_{i} \cdot h\right)$

are 0 and $h$ themselves. Geometrically, the set of neighbors are those lattice points $h$ such that $(0, h)$ is an edge of $K(A)$.

The complex $K Z(A)$ is intimately related to what is known as the Frobenius problem: Let $p=\left(p_{0}, \ldots, p_{n}\right)$ be a vector of positive integers whose greatest common divisor is unity. The Frobenius problem is to find the largest integer $f^{*}$ which cannot be written as a nonnegative integral combination of the $p_{i}$. Let $A$ be a matrix of size $(n+1) \times n$, whose columns generate the $n$ dimensional lattice of integers $h$ satisfying $p \cdot h=0$. Then it has been shown (Scarf and Shallcross, 1992) that

$$
f^{*}=\max (p \cdot b \mid A x \leqslant b \text { is a maximal lattice free-body })-\sum p_{i} .
$$

Since all maximal lattice free bodies $(x \mid A x \leqslant b)$ are similar in shape, the Frobenius number can be obtained by finding that maximal lattice free body with maximal volume.

\section{A geometric realization of $K(A)$}

We now begin the process of proving Theorems 1 and 2. Consider the mapping of $\mathbb{R}^{n}$ into $\mathbb{R}^{n+1}$ given by

$$
y_{i}=\exp \left(t a_{i} \cdot x\right) \text { for } i=0, \ldots, n,
$$

with $t$ a fixed positive real number. Clearly the image of $\mathbb{R}^{n}$ under this mapping is contained in the set $M=\left(y=\left(y_{0}, \ldots, y_{n}\right) \mid y_{i}>0, \Pi y_{i}=1\right)$ and, as we shall see, the image is the entire sheet of this hyperboloid. (Scarf, 1973, contains an earlier but less useful version of this mapping.)

Let $V=(v)$ be the image of the lattice of integers $Z^{n}$. Notice that $V$ is a discrete set, i.e., every compact set in $\mathbb{R}^{n+1}$ contains finitely many elements of $V$. We can define a multiplication in $\mathbb{R}^{n+1}$ by taking the coordinate-wise product of two vectors. With this multiplication, $M$ is an Abelian group with identity $1=(1, \ldots, 1)$, and $V$ a discrete subgroup. Finally, let $C$ be the convex hull of $V$. (For simplicity of notation, we omit explicit reference to the dependence of $V, C$ and other constructions on the parameter $t$.)

$C$ is not the convex hull of finitely many points and so it need not be, and is not, a convex polytope. We can, nevertheless, define faces of $C$ in the following way. $L$ is a face of $C$ if there is a half-space $H \subset \mathbb{R}^{n+1}$ with bounding hyperplane $H^{0}$ such that $C \subset H$ and $C \cap H^{0}=L$. The vertices, or zero dimensional faces of $C$, are precisely the vectors $v \in V$, since each such vector is on the boundary of the strictly convex body $\left(y \mid y>0, \Pi y_{i} \geqslant 1\right)$. The line segment $[v, u]$ will be an edge of $C$ (a one dimensional face) if and only if the segment $\left[1, v u^{-1}\right]$ is also an edge. More generally the facial structure of $C$ is identical at each vertex; the convex hull of $\left(u, v^{1}, \ldots, v^{k}\right)$ will be a face of $C$ if and only if the convex hull of $\left(1, v^{1} u^{-1}\right.$, $\ldots, v^{k} u^{-1}$ ) is also a face of $C$. (If $\left(y: \sum h_{i} y_{i} \geqslant h\right.$ ) is the half-space which verifies that the first convex hull is a face, then $\left(y: \sum h_{i} u_{i} y_{i} \geqslant h\right)$ verifies that the second convex hull is a face, 
and conversely). As we shall see (Theorem 3), there is a $t_{0}$ such that for $t>t_{0}$, the facets or faces of dimension $n$ - of $C$ are simplices and the number of facets containing any particular vertex is finite. As a consequence, $C$ will have the facial structure of a polytope at each vertex.

In order to prove Theorem 1 we shall require some preliminary arguments. We start with three lemmas concerned with the facial structure of $C$.

Lemma 1. The image of $\mathbb{R}^{n}$ under the mapping (2) is the entire set $M=\left(y \mid y>0, \Pi y_{i}=1\right)$.

Proof. Let $y$ be an arbitrary point in $M$. The $n \times n$ minors of $A$ are non-singular so that there is a solution, $x$, to the system of equations

$$
a_{i} \cdot x=(1 / t) \log y_{i}, \quad \text { for } i=1, \ldots, n .
$$

Since $\Pi y_{i}=1$, the same $x$ satisfies the corresponding equation for $i=0$.

Lemma 2. Let $\epsilon>0$ be given. Then for each $i$, there is $a v \in V$ with $v_{j} \leqslant \epsilon$ for all $j \neq i$.

Proof. Take $i=0$. From (A1), the set $\left(x \mid a_{j} \cdot x \leqslant(1 / t) \log \epsilon, j=1, \ldots, n\right)$ is a cone with nonempty interior and therefore contains a lattice point. The argument for other $i$ is similar.

Lemma 3. Let $y^{0}$ be on the boundary of C and let $h=\left(h_{0}, \ldots, h_{n}\right) \neq 0$ be such that $h \cdot y \geqslant h \cdot y^{0}$ for all $y \in C$. Then $h_{i}>0$ for all $i$.

Proof. $y^{0}$ is in the set $\left(y \mid y>0, \Pi y_{i} \geqslant 1\right)$, so that all of its coordinates are strictly positive. If one of the coordinates of $h$, say $h_{i}$, were $<0$, then, as $\epsilon$ tends to zero, the vectors $v$ described in Lemma 2 will lie in $C$ and violate the inequality $h \cdot v \geqslant h \cdot y^{0}$. It follows that $h_{i} \geqslant 0$, and that $h \cdot y^{0}$ is strictly positive since the coordinates of $h$ are $\geqslant 0$, but not all zero, and $y^{0}>0$. But then if $h_{i}=0$, these same vectors in $V$ will violate $h \cdot v \geqslant h \cdot y^{0}$.

We now turn to three lemmas concerning the structure of $K(A)$.

Lemma 4. Each lattice point $z \in Z^{n}$ is contained in a finite number of simplices in $K(A)$.

Proof. Let the lattice point be $z=0$, with simplex $\left(0, z^{1}, \ldots, z^{n}\right)$, associated with the maximal lattice free body given by $(x \mid A x \leqslant b)$, where $b_{i}=\max _{j} a_{i} \cdot z^{j} \geqslant 0$. Any particular set of this form contains a finite number of lattice points; if there were an infinite sequence of such maximal lattice free bodies, all differing from each other, then in this sequence one of the coordinates, say $b_{0}$, would tend to infinity. But this is impossible since the cone defined by $a_{i} \cdot x \leqslant 0$, for $i=1, \ldots, n$, has a non-empty interior which contains infinitely many lattice points.

$N(0)$, the set of neighbors of the origin, is identical with the set of $\left(z^{i}-z^{j}\right)$, with $z^{i}$ and 
$z^{j}$ vertices of a common simplex in $K(A)$. We note that Lemma 4 implies that $N(0)$ is finite. Lemma 4 has another useful implication.

Lemma 5. Define $\delta_{1}=\min \left(\left|a_{i} \cdot h\right|: h \in N(0), i=0,1, \ldots, n\right)$. Let $S$ be a finite set of lattice points and let $K=(x \mid A x<b)$, where $b_{i}=\max \left(a_{i} \cdot z: z \in S\right)$. Then if $K$ contains a lattice point, it will contain a lattice point $\xi$ with $a_{i} \cdot \xi \leqslant b_{i}-\delta_{1}$ for all $i$.

Proof. Let $\xi \in K$ and let $i$ be an index such that $b_{i}-\delta_{1}<a_{i} \cdot \xi<b_{i}=a_{i} \cdot z^{i}$ with $z^{i}$ a particular lattice point in $S$. Then $\xi$ and $z^{i}$ are not neighbors. It follows that the smallest body of the form $(x \mid A x \leqslant c)$ containing $\xi$ and $z^{i}$, will also contain an interior lattice point. If the conclusion of Lemma 5 is not valid for this interior lattice point in this smaller body - which is strictly contained in $K$-we repeat the argument. But the argument must terminate after a finite number of repetitions, since each repetition eliminates at least one interior lattice point.

We have one final Lemma before proceeding to the proof of Theorem 1.

Lemma 6. There is a $\delta_{2}>0$, such that if $\left(z^{0}, z^{1}, \ldots, z^{n}\right)$ is a simplex in $K(A)$, ordered so that for each $i, \max _{j} a_{i} \cdot z^{j}$ is assumed at $z^{i}$, and if $z$ is a lattice point different from $z^{0}, z^{1}, \ldots$, $z^{n}$, then $a_{i} \cdot z \geqslant a_{i} \cdot z^{i}+\delta_{2}$ for some $i$.

Proof. It is sufficient to demonstrate Lemma 6 for those simplices in $K(A)$ with $z^{0}=0$, since the general simplex may be reduced to this case by subtracting $z^{0}$ from each $z^{i}$. The number of such simplices is finite and it is therefore sufficient to find a $\delta_{2}$ for each simplex and take the smallest of these. But, of course, there is such a $\delta_{2}$ for any particular simplex $\left(0, z^{1}, \ldots, z^{n}\right)$ in $K(A)$, since otherwise there would be an infinite set of lattice points, $z(\epsilon)$, satisfying $a_{i} \cdot z(\epsilon)<a_{i} \cdot z^{i}+\epsilon$, for $i=0, \ldots, n$, and $\epsilon$ tending to zero.

\section{The proof of Theorem 1}

Theorem 1 will be demonstrated by means of the following result, which shows that for large $t$, the convex hulls of the images of the vertices of simplices in $K(A)$ provide a geometric realization of the complex.

Theorem 3. There is a $t_{0}$ such that for each $t>t_{0}$, the $n$-facets of $C$ are simplices. Moreover, for such $t$, the simplex $\left(z^{0}, z^{1}, \ldots, z^{n}\right) \in K(A)$ if and only if the images of its vertices $\left(v^{0}\right.$, $\left.v^{1}, \ldots, v^{n}\right)$ are the vertices of an $n$-facet of $C$.

Proof. The proof consists of two parts. We begin by letting $\left(z^{0}, z^{1}, \ldots, z^{n}\right)$ be an arbitrary set of $n+1$ lattice points whose images $\left(v^{0}, v^{1}, \ldots, v^{n}\right)$ lie on an $n$-facet of $C$, for some 
particular $t>t_{1}=(\log (n+1)) / \delta_{1}$, and demonstrate that these $n+1$ lattice points form a simplex in $K(A)$.

Let $h$ be the strictly positive normal to the hyperplane supporting the facet containing $\left(v^{0}, v^{1}, \ldots, v^{n}\right)$, for the particular $t$ in question. We have

$$
\sum h_{i} \exp \left(t a_{i} \cdot z\right) \geqslant 1,
$$

for any lattice point $z$, with equality for $z=z^{0}, \ldots, z^{n}$.

Consider first $z=z^{j}$. It follows from the above equality that for each index $i$,

$$
h_{i} \exp \left(t a_{i} \cdot z\right) \leqslant 1,
$$

so that

$$
a_{i} \cdot z^{j} \leqslant-(1 / t) \log \left(h_{i}\right),
$$

and therefore

$$
\max _{j}\left[a_{i} \cdot z^{j}\right] \leqslant-(1 / t) \log \left(h_{i}\right) .
$$

We wish to show that there are no lattice points other than $z^{0}, z^{1}, \ldots, z^{n}$ in the body $(x \mid A x \leqslant b)$, with $b_{i}=\max _{j}\left[a_{i} \cdot z^{j}\right]$, so that $\left(z^{0}, z^{1}, \ldots, z^{n}\right)$ is a simplex in $K(A)$. Let $z$ be such a lattice point $\left(z=z^{j}\right.$ is possible at the moment) so that $a_{i} \cdot z \leqslant \max _{j}\left[a_{i} \cdot z^{j}\right]-\delta_{1}$, for all $i$, using Lemma 5. But then inequality (3) implies that there is an index $i$ with

$$
h_{i} \exp \left(t a_{i} \cdot z\right) \geqslant 1 /(n+1),
$$

and

$$
\begin{gathered}
a_{i} \cdot z \geqslant-(1 / t) \log \left(h_{i}\right)-(1 / t) \log (n+1) \\
\geqslant \max _{j}\left[a_{i} \cdot z^{j}\right]-(1 / t) \log (n+1),
\end{gathered}
$$

a contradiction if $t>t_{1}=(\log (n+1)) / \delta_{1}$.

We now turn to the second part of the argument and demonstrate that the convex hull of the images of the vertices of a simplex $\left(z^{0}, z^{1}, \ldots, z^{n}\right)$ in $K(A)$ form a facet of the boundary of $C$ for large $t$. Let $\left(v^{0}, v^{1}, \ldots, v^{n}\right)$ be such a set of images and let $h$ satisfy $h \cdot v^{j}=1$, for $j=0,1, \ldots, n$. We will demonstrate the existence of a $t_{2}$, for this simplex, such that $h \cdot v \geqslant 2$, for all $t \geqslant t_{2}$, and for all $v$ which are images of lattice points other than $z^{j}$. Assume that the vertices have been permuted so that $a_{i} \cdot z^{i}=\max _{j}\left[a_{i} \cdot z^{j}\right]$.

By Cramer's rule we have

$$
h_{0}=\operatorname{det} N / \operatorname{det}\left[\exp \left(t a_{i} \cdot z^{j}\right)\right]
$$

with $N$ the matrix obtained by replacing row 0 by $(1, \ldots, 1)$ in the matrix appearing in the denominator. Let us estimate the denominator first. The determinant can be written as the sum of $(n+1)$ ! terms, each one based on a permutation of $(0,1, \ldots, n)$. But for each permutation, $\sigma$, other than the identity, the corresponding term will be $\left[\Pi \exp \left(a_{i} \cdot z^{\sigma(i)}\right)\right]^{t}$, which is strictly less than

$$
\left.\Pi \exp \left(a_{i} \cdot z^{i}\right)\right]^{t},
$$


so that for large $t$ this single term will be the asymptotic value of the determinant in the denominator. A similar analysis tells us that the numerator is asymptotically equal to the same product with index ranging from 1 to $n$, so that $h_{0} \sim \exp \left(-t a_{0} \cdot z^{0}\right)$, in the sense that

$$
h_{0}=\left(1+\epsilon_{0}(t)\right) \exp \left(-t a_{0} \cdot z^{0}\right), \quad \text { with } \epsilon_{0}(t) \rightarrow 0 \text { as } t \rightarrow \infty .
$$

By a similar argument we obtain

$$
h_{i}=\left(1+\epsilon_{i}(t)\right) \exp \left(-t a_{i} \cdot z^{i}\right), \text { with } \epsilon_{i}(t) \rightarrow 0 \text { as } t \rightarrow \infty \text {, for all } i .
$$

In particular there is a $t_{2}$ so that for all $t \geqslant t_{2}$, we have

$$
h_{i} \geqslant 2 \exp \left(-t a_{i} \cdot z^{i}\right) \exp \left(-t \delta_{2}\right) \text { for all } i,
$$

with $\delta_{2}$ the constant referred to in Lemma 6.

In order to demonstrate that $\operatorname{con}\left(v^{0}, v^{1}, \ldots, v^{n}\right)$ is a facet of the boundary of $C$, we shall show that $h \cdot v \geqslant 2$, for $t \geqslant t_{2}$, and for any image, $v$, of a lattice point other than $\left(z^{j}\right)$. From (4), we have

$$
h \cdot v \geqslant 2 \sum \exp \left(t\left(a_{i} \cdot z-a_{i} \cdot z^{i}-\delta_{2}\right)\right) \geqslant 2,
$$

for any integral $z$ other than $\left(z^{j}\right)$, since, for that $z$, Lemma 6 states that there must be at least one index $i$ with $a_{i} \cdot z \geqslant a_{i} \cdot z^{i}+\delta_{2}$.

In this argument, the value of $t_{2}$ depends on the particular simplex $\left(z^{0}, z^{1}, \ldots, z^{n}\right)$; in order to complete the proof of Theorem 1 we must show that a single value suffices for all simplices. But for any $t$, the image of $\left(z^{0}, z^{1}, \ldots, z^{n}\right)$ is a facet of $C$ if the image of $(0$, $\left.z^{1}-z^{0}, \ldots, z^{n}-z^{0}\right)$ is a facet. There is a finite number of simplices in $K(A)$ for which $z^{0}=0$, and we may select the largest value of $t_{2}$ associated with any of these.

The value of $t_{0}$ in the statement of Theorem 3 is the larger of $t_{1}$ and $t_{2}$. It should be remarked that we have demonstrated that for $t>t_{0}$, the $n$-facets of $C$ are simplices. For if the convex hull of $\left(v^{0}, v^{1}, \ldots, v^{n}\right)$ is contained in an $n$-facet of $C$, we know that its preimage $\left(z^{0}, z^{1}, \ldots, z^{n}\right)$ is a simplex in $K(A)$ and, consequently, the convex hull of $\left(v^{0}, v^{1}, \ldots, v^{n}\right)$ is, itself, an $n$-facet.

Theorem 3 yields a geometric realization of the complex $K(A)$ as the boundary of the convex set $C$, for $t>t_{0}$ in the following fashion: given a particular simplex in $K(A)$ with vertices $\left(z^{0}, z^{1}, \ldots, z^{n}\right)$, associate the point $x=\sum \alpha_{j} v^{j}$ with the formal convex combination $\sum \alpha_{j} z^{j}$, for each $\alpha=\left(\alpha_{0}, \ldots, \alpha_{n}\right)$ with $\alpha_{j} \geqslant 0, \sum \alpha_{j}=1$. With this mapping, $F:|K(A)| \rightarrow \partial C$, vectors arising from different simplices in $K(A)$ or from the same simplex but with different formal convex combinations, are mapped to different points on the boundary of $C$. Thus $F$ provides a homeomorphism between $|K(A)|$ and $\partial C$, and Theorem 1 follows from the observation that $\partial C$ is homeomorphic to $\mathbb{R}^{n}$.

For the proof of Theorem 2, we need an argument from equivariant topology which we shall state in a somewhat informal way. Consider the composite mapping obtained by first mapping the formal convex combination $\sum \alpha_{j} z^{j}$ to $x$ on the boundary of $C$, as above, and then mapping $x$ to $y$ in $M$, by $y_{i}=x_{i} /\left[\Pi x_{j}\right]^{1 / n}$. This composite mapping, $T:|K(A)| \rightarrow M$, 
is a realization of the complex $K(A)$ as the hyperboloid $M$. Furthermore the mapping $T$ is compatible with (equivariant for) the actions of $Z^{n}$ on $|K(A)|$ (by translation) and on $M$ (by coordinatewise multiplication by points in $V)$, i.e., if $\left(y_{0}, \ldots, y_{n}\right)$ is the coordinate vector associated with the formal convex combination $\sum \alpha_{j} z^{j}$, then the coordinate vector associated with its lattice translate $\sum \alpha_{j}\left(z^{j}+z\right)$ is $\left(y_{0} v_{0}, \ldots, y_{n} v_{n}\right)$, with $v$ the image of $z$ under the mapping (2). Therefore, the set of lattice translates of a given point in $|K(A)|$ corresponds to a single point in $M / V$, so that $T$ induces a homeomorphism between $|K Z(A)|=|K(A)| / Z^{n}$ and the quotient space $M / V$. Theorem 2 follows from the observation that $M / V$ is homeomorphic to the $n$-torus $\mathbb{R}^{n} / Z^{n}$.

\section{Acknowledgement}

The authors are extremely grateful to William Massey and Xin Wang for many clarifying conversations.

\section{References}

W.S. Massey, Singular Homology Theory (Springer-Verlag, New York, 1980).

H. Scarf, The Computation of Economic Equilibria (with collaboration of Terje Hansen), Cowles Foundation Monograph No. 24 (Yale University Press, New Haven, 1973).

H. Scarf, "Production sets with indivisibilities, Part I: Generalities," Econometrica 49 (1) (1981a) 1-32.

H. Scarf, "Production sets with indivisibilities, Part II: The case of two activities," Econometrica 49 (2) (1981b) $395-423$.

H. Scarf, "Integral polyhedra in three space," Mathematics of Operations Research 10 (3) (1985) $403-438$.

H. Scarf, "Neighborhood systems for production sets with indivisibilities," Econometrica 54 (3) (1986) 507532.

H. Scarf and D. Shallcross, "Shortest integer vectors," Mathematics of Operations Research 18 (3) (1993) 516522.

P. White, "Discrete activity analysis," Ph.D. Thesis, Yale University, Department of Economics (1983). 